\title{
Il faut plus de femmes scientifiques dans les médias! Journée Sciences et Médias 2021
}

Cécile Michaut (www.scienceetpartage.fr)

Docteure en chimie, journaliste scientifique et formatrice en vulgarisation

Organismes de recherche et journalistes doivent travailler ensemble pour une meilleure représentation des femmes dans les médias. Au-delà de l'équité, cette diversité est garante d'une meilleure science.

Après deux reports, la cinquième édition des Journées Sciences et Médias : « Femmes scientifiques à la Une ! Comment améliorer la représentation des femmes scientifiques dans les médias ? " s'est enfin tenue le 29 janvier 2021 à la Bibliothèque nationale de France $(\mathrm{BnF}$, site François Mitterand, Paris $13^{\mathrm{e}}$ ).

Cette journée était coorganisée par la BnF, I'Association des Journalistes Scientifiques de la Presse d'Information (AJSPI), la Société Française de Physique (SFP), la Société Chimique de France (SCF), la Société Informatique de France (SIF), la Société de Mathématiques Appliquées et Industrielles (SMAI) et la Société Mathématique de France (SMF).

Site des journées Science et Médias et accès aux vidéos en ligne des interventions de la journée www.sciencesetmedia.org
$\mathrm{P}$ andémie de Covid-19 oblige, le public suivait les débats à distance. Mais les intervenants étaient bien présents, et le public interagissait via les réseaux sociaux, par l'entremise d'Agnès vernet de l'AJSPI.

\section{Mieux travailler ensemble}

Daniel Hennequin, président de la commission Culture scientifique de la SFP, l'une des " chevilles ouvrières » de cette journée selon les mots de l'animateur Mathieu Rouault (journaliste à Grand Labo), rappelle le but de ces journées « Sciences et Médias »: "En travaillant sur le lien entre la science et la société, nous découvrons les visions respectives côté médias comme côté science et cherchons des solutions communes. Cela se reflète dans le programme de la journée."

Audrey Mikaëlian, vice-présidente de l'AJSPI, pose la question clé : pourquoi faut-il plus de femmes en sciences ? "Ce n'est pas seulement une question de justice, d'équité et de diversité, souligne-t-elle. Au-delà de la lutte contre les discriminations, il faut plus de femmes dans les sciences pour améliorer la qualité même de la science. La diversité des humains permet celle des points de vue intellectuels. " Mais on ne parle pas aujourd'hui que de sciences, on parle de médias. Qu'est-ce que cela implique d'être visible par des millions de lecteurs ou téléspectateurs? "Cette visibilité, c'est du pouvoir, affirme Audrey Mikaëlian. Celui de convaincre, de faire avancer ses idées. " C'est pour cela que les femmes doivent être plus visibles. Elles ne sont pas les petites mains d'une science décidée par les hommes. 


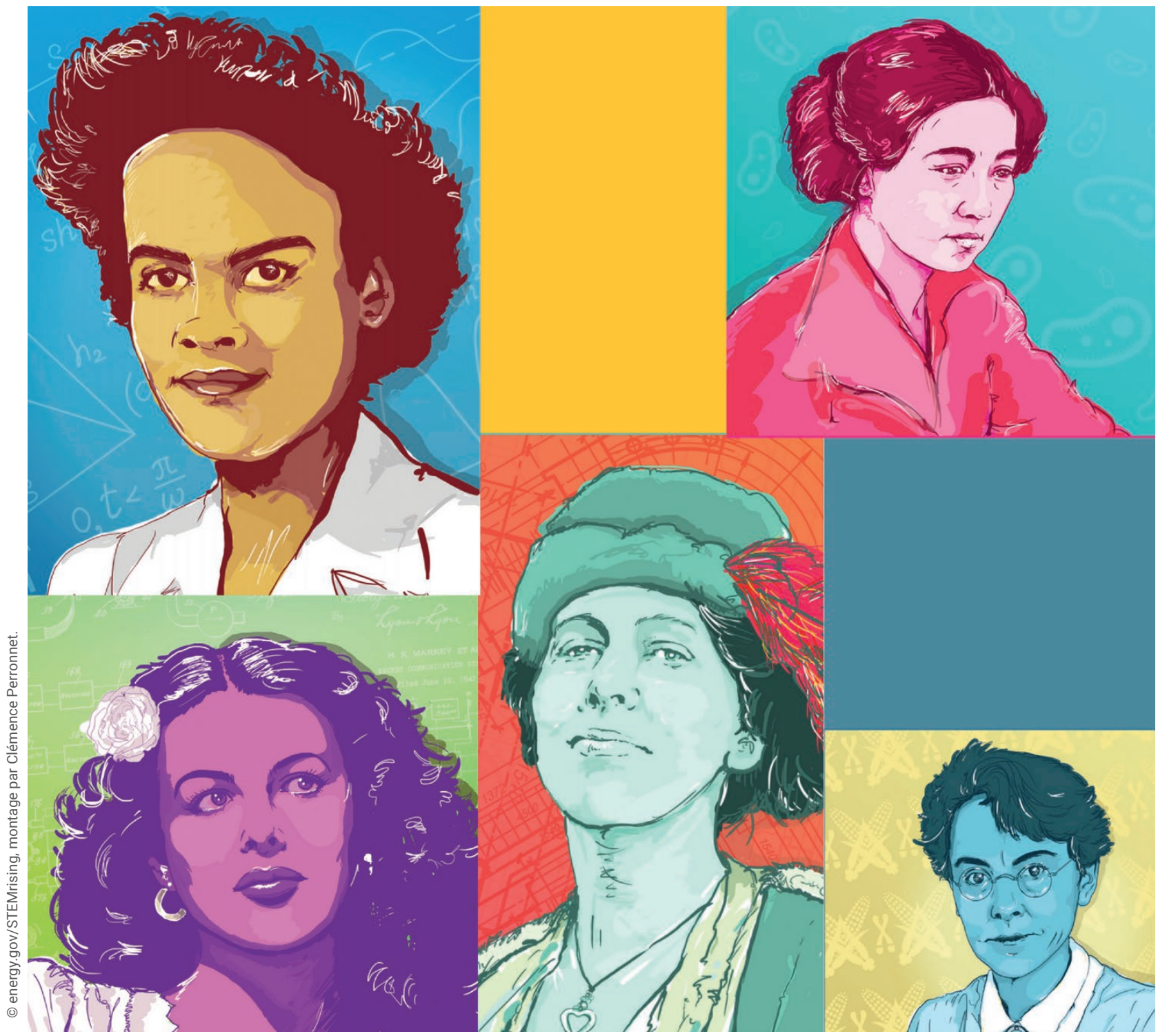

Portraits de quelques femmes scientifiques américaines (USA). De gauche à droite, puis de haut en bas : Evelyn Boyd Granville (1924-), mathématicienne afro-américaine qui a participé au programme Apollo de la NASA. Ruby Hirose (1904-1960), biologiste et biochimiste qui a travaillé sur les vaccins contre la poliomyélite. Hedy Lamarr (1914-2000), coïnventrice d'un système de communication secrète pour engins téléguidés. Nora Stanton Blatch Barney (1883-1971), première femme ingénieure civile aux USA. Barbara McClintock (1902-1992), première femme prix Nobel de physiologie et médecine pour la découverte de nouveaux facteurs génétiques.

\section{«Quand c'est sérieux, place aux hommes »}

Céline Calvez, députée des Hauts-de-Seine, coordinatrice LREM au sein de la Commission des affaires culturelles et de l'éducation de l'Assemblée nationale, souligne la baisse du nombre des expertes dans les médias lors d'une crise comme celle du Covid-19: $9 \%$ seulement d'expertes interviewées dans le journal télévisé d'une grande chaine en mars 2020 contre 35 à $40 \%$ en temps normal, $84 \%$ de personnalités masculines à la une des sept grands quotidiens nationaux entre mars et fin mai 2020. "Comme si, les choses devenant sérieuses, les femmes devaient s'effacer au profit des hommes ", observe la députée. Son rapport du 15 septembre 2020 sur la place des femmes dans les médias en temps de crise [1] contient vingt-six préconisations, parmi lesquelles « Compter la présence des femmes : dans quel type d'émissions sont-elles ? Sur quels sujets interviennent-elles ?
Sont-elles davantage interrompues ? " Les médias pourront effectuer ces comptages grâce à des outils automatisant cette tâche, par exemple en reconnaissant les voix d'hommes ou de femmes par intelligence artificielle. Bel apport de la science à l'égalité !

\section{Dans la presse écrite, un massif manque de parité}

En attendant ces outils, il faut compter... à la main. C'est ce qu'a fait Cécile Michaut, journaliste scientifique, qui a analysé sept journaux durant le mois de janvier 2021 (fig. 1): quatre mensuels scientifiques (Sciences et Avenir, Science \& Vie, Pour la Science et La Recherche) et trois quotidiens nationaux (Le Monde, Le Figaro, Libération). Objectif : dénombrer le nombre de chercheurs et chercheuses cité.e.s et mis·e.s en valeur par des portraits, des interviews... 
$>>$

Aucun journal n'est paritaire, certains allant jusqu'à $80 \%$ d'hommes cités. Les journaux qui, comme La Recherche et Pour la Science, publient des articles écrits par des scientifiques, choisissent majoritairement des auteurs plutôt que des autrices. Du côté de la presse nationale, même constat. Et si Le Monde cite $35 \%$ de femmes scientifiques dans son cahier hebdomadaire "Sciences", ses pages sur le coronavirus sont bien plus masculines. Lors d'une crise, on demande aux femmes de s'effacer. Les chiffres moyens de 3/4 d'hommes pour $1 / 4$ de femmes se retrouvent dans Libération et Le Figaro.

Le fait que le manque de parité soit général et massif indique que le problème n'est pas dû à quelques journalistes : il est systémique. C'est donc au niveau de la direction des rédactions que cette question doit être prise en compte. Et tout démarrera par des comptages : sans données, on ne peut pas agir.

\section{Nommer les savantes au féminin}

Parler des femmes de sciences, c'est aussi examiner comment elles sont nommées. C'est le travail d'éliane viennot, professeuse de littérature. Nommer les savantes avec les mots qui conviennent est indispensable pour construire une société égalitaire. Elle rappelle que les termes féminins rejetés s'appliquent à des domaines prestigieux : le savoir, la pensée, la création, le leadership.

Aucune réticence à nommer au féminin les boulangères, les coiffeuses ou les caissières. Ce n'est donc pas un manque de matériel linguistique. On peut dire autrice aussi bien qu'actrice, doctoresse aussi bien que princesse, écrivaine aussi bien que contemporaine, médecine aussi bien que voisine, professeuse aussi bien que chanteuse, chirurgienne aussi bien que musicienne. Ce n'est pas la langue qui " bugge », ce sont nos têtes. En sciences, l'absence des mots adéquats (doctoresse, médecine, professeuse) mesure la fragilité des positions que les femmes occupent dans ce bastion masculin.

De nombreuses universités refusent de féminiser le mot « docteur ». Or, ce mot est emblématique : le doctorat est le grade le plus élevé de tous. "On est là au cœur de la forteresse, qui s'est occupée dès sa naissance d'exclure les femmes des bienfaits de la science", souligne Éliane Viennot.

Celles et ceux qui croient ce combat dérisoire se trompent. "Dans une société où personne n'ose plus s'opposer ouvertement à l'égalité des sexes, des salaires, de l'accès à l'instruction, c'est par d'autres voies, comme le langage, que s'exprime le refus d'aller plus loin dans la construction d'un avenir égalitaire", conclut Éliane Viennot. Dans cette lutte sur les mots, le rôle des médias est crucial.

\section{La culture scientifique se décline au masculin}

Clémence Perronnet, chercheuse en sociologie et maitresse de conférences en sciences de l'éducation, s'est intéressée aux produits culturels scientifiques destinés à la jeunesse. Elle a analysé quatre catégories de produits culturels : ceux qu'on peut visiter (musées, CCSTI, expositions), ceux qu'on peut lire (sur papier ou écran), ceux qu'on peut regarder et écouter, et enfin ceux qui permettent de pratiquer : les jeux et activités en associations.

Les femmes de science sont presque invisibles partout. Les rares figures féminines sont souvent porteuses de stéréotypes : passives, voire décoratives, cantonnées à des domaines comme le soin.

Quelques exemples : à Universcience (Cité des Sciences, Palais de la découverte), dans une exposition permanente sur la lumière, "il $\gamma$ avait autant de femmes que de portraits d'Albert Einstein " sourit (jaune) Clémence Perronnet. La plupart de ces femmes sont représentées non comme des actrices, mais comme des objets d'expériences scientifiques. Enfin, les voix féminines donnent des consignes, les masculines transmettent le contenu scientifique.

Les manuels scolaires ne donnent à voir que 3\% de femmes scientifiques. Seuls des hommes sont montrés en train de pratiquer des sciences. Dans une encyclopédie sur le corps, la seule fille de l'ouvrage était sur la page « et si nous n'avions pas de cerveau »! " Ces livres pour la jeunesse véhiculent des propos sexistes, et participent à la justification de l'ordre inégal et sexiste", analyse Clémence Perronnet.

Dans la presse, même constat. À Science \& Vie Junior, une enquête en 2013 montrait qu'en neuf ans, pas une femme n'avait fait seule la couverture du magazine !

À l'écran aussi, les femmes sont les grandes absentes. Une femme pour sept hommes scientifiques au cinéma, $87 \%$ d'hommes scientifiques dans les dessins animés, ou encore $71 \%$ d'hommes dans l'émission de télévision très appréciée « C'est pas sorcier » (fig. 2).
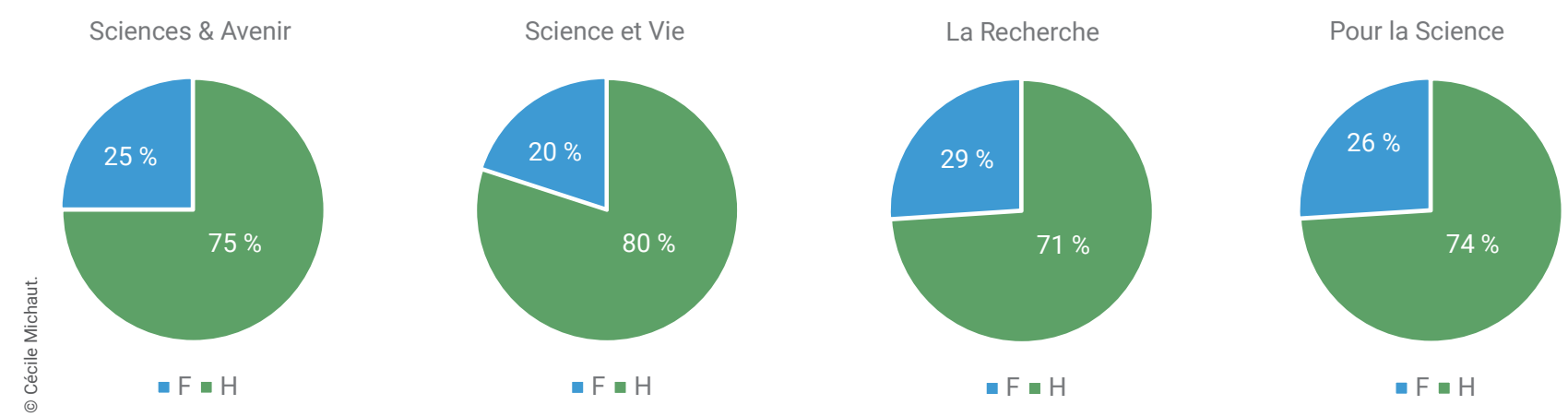

1. Proportions de femmes (en bleu) et d'hommes (en vert) cités dans quatre mensuels scientifiques 


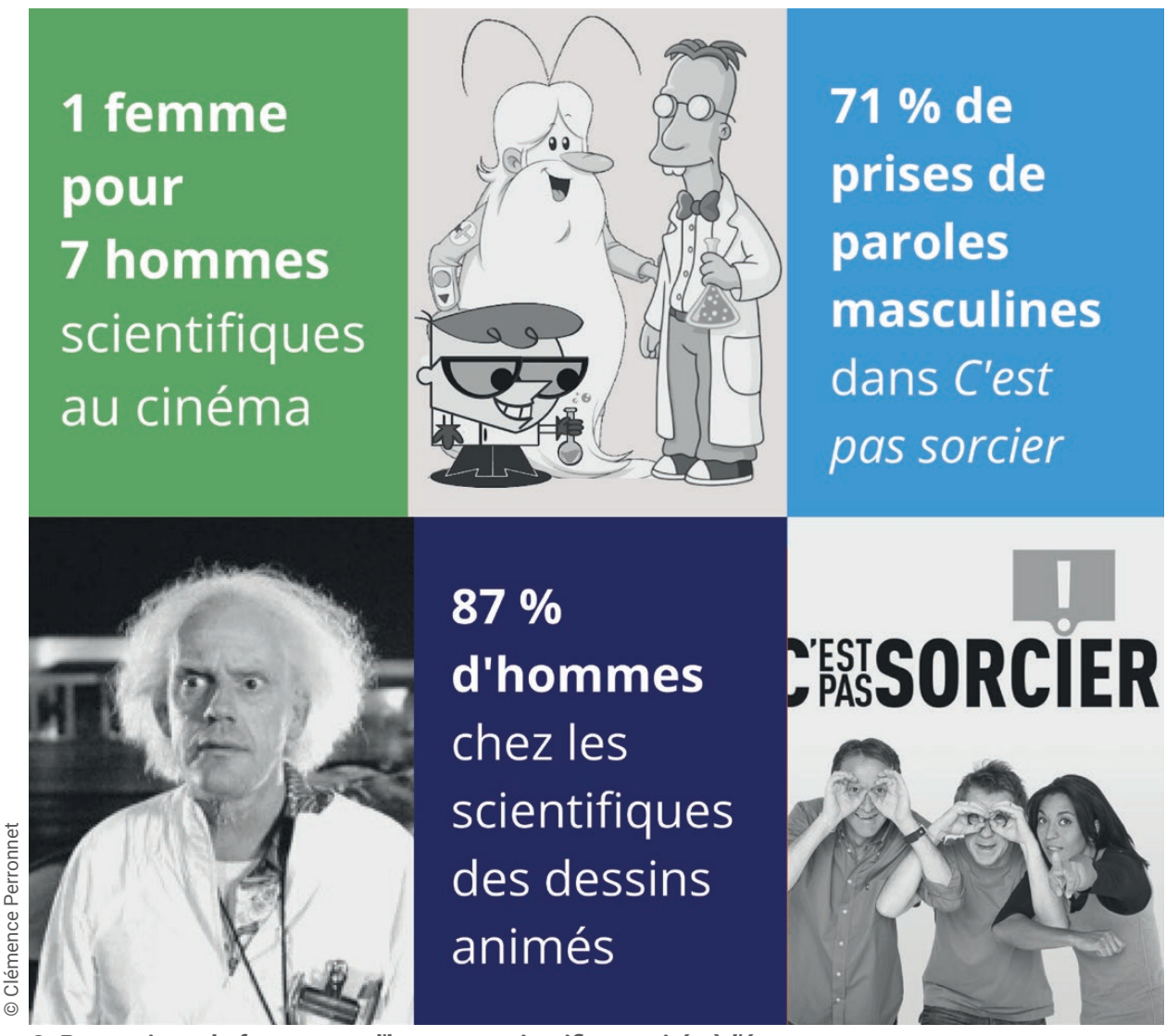

2. Proportions de femmes et d'hommes scientifiques cités à l'écran.

\section{En presse : compter pour réaliser}

Place à la première table ronde sur les bonnes pratiques dans les médias, avec Audrey Mikaëlian, Philippe Pajot, rédacteur en chef de La Recherche, et Pascaline Minet, cheffe de la rubrique "Sciences " dans le quotidien généraliste suisse Le Temps.

"À La Recherche, les comptages entre 2017 et 2019 nous ont surpris, on pensait que c'était plus paritaire, souligne Philippe Pajot. Il faut agir : c'est une question d'éthique et de crédibilité du journal. " Pour Audrey Mikaëlian, la diversité doit être partout : l'âge, l'origine... " Un doctorant a un point de vue différent d'un ponte. Les femmes ne sont pas élevées de la même façon et ont donc un point de vue différent."

Au Temps, depuis 2018, un baromètre de la parité permet de suivre la représentativité des femmes dans le journal. "On regarde l'évolution et, malheureusement, en sciences, on stagne autourde $30 \%$ » observe Pascaline Minet. À la télévision, "il n'y a aucune demande des chaines en matière de parité " indique Audrey Mikaëlian.

Idem, côté jeux et jouets. En 2018, la marque leader du marché proposait 56 coffrets science avec un garçon, pour seulement onze avec garçon et fille, ou fille seule. Les stéréotypes demeurent : expérience, espace, construction, dinosaure pour les garçons, nature et chimie pour les filles.

On a observé cependant quelques progrès ces dernières années. Par exemple, le retour après dix ans d'absence d'une jeune fille en couverture de Science \& Vie Junior, le succès du film "Les figures de l'ombre ", ou la création de personnages féminins de la Nasa dans des jeux Lego. C'est crucial : "dans mon travail auprès des enfants, j'ai vu à quel point quelques exemples suffisent à transformer les imaginaires en formation ", témoigne Clémence Perronnet.

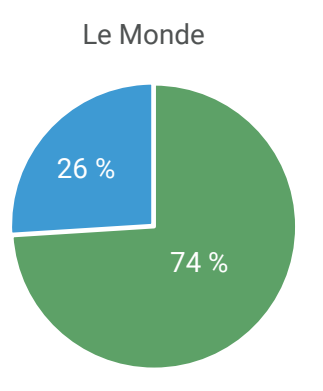

$\square \mathrm{F}=\mathrm{H}$
Le Figaro

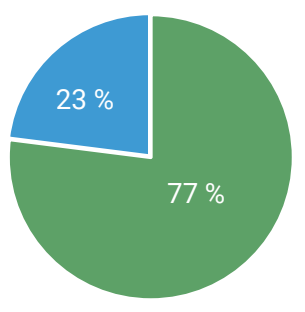

$\square \mathrm{F} \square \mathrm{H}$
Libération

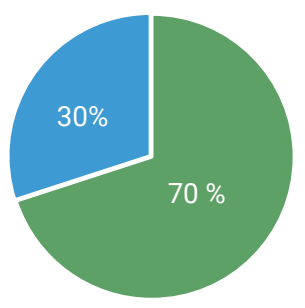

$\square \mathrm{F} \square \mathrm{H}$
Donner plus de places aux femmes dépend aussi du temps dont on dispose : dans l'urgence, on prend la personne disponible. Il faut beaucoup de sensibilisation pour que les journalistes aient la parité en tête au quotidien. Un système d'alerte automatique pour signaler qu'un article ne comporte pas assez d'interlocutrices peut aider les journalistes à être vigilants.

L'écriture " non genrée " est souvent simple à mettre en œuvre, par exemple "Cro-Magnon " plutôt que " homme de Cro-Magnon ". Côté écriture inclusive, " on utilise cheffe, autrice, doctoresse, mais on ne met pas les points médians car cela braque les lecteurs ", observe Pascaline Minet. Valoriser les femmes, c'est aussi écrire sur elles. "Montrer plus de femmes scientifiques leur donnera plus envie de s'exprimer, c'est un cercle vertueux à mettre en place, et pour cela il faut être volontariste", conclut Pascaline Minet.

\section{Des femmes à la tête des médias}

Et si la sous-représentation de femmes aux postes de direction influençait les contenus ? C'est l'hypothèse à l'origine de l'association de femmes journalistes « Prenons la une ", représentée par sa présidente Léa Lejeune.

Dans les rédactions qui ont pris des décisions concrètes, les progrès sont notables. Le meilleur élève est France Télévision, dirigé par Delphine Ernotte, qui est passé de 25 à $40 \%$ de femmes expertes dans les débats télévisés. "Cela montre que c'est possible lorsqu'il $\gamma$ a une politique volontariste » constate Léa Lejeune. Mais ce chiffre est retombé à $9 \%$ lors du premier confinement ! En temps de crise, les bonnes pratiques passent à la trappe. 


\section{6 || faut que les femmes soient visibles à hauteur du pourcentage de femmes que l'on vise et non de l'existant."}

\begin{abstract}
Parmi les freins, le mythe du «bon client médiatique ": quelqu'un de disponible, qui s'exprime clairement et brièvement. Pris dans une course au temps, les médias font appel à ces mêmes personnes. Inversement, trop de chercheuses ne se sentent pas légitimes. Les journalistes pressés appellent alors quelqu'un d'autre. Il faudrait plutôt qu'ils accompagnent et rassurent cette experte sur sa légitimité.

Léa Lejeune propose de former les chercheuses à la prise de parole dans les médias, et de former à la parité dans les écoles de journalisme. Bien sûr, inciter les chercheuses à s'inscrire sur le guide (numérique et gratuit) Les expertes [2]. "Chercheuses et journalistes femmes, nous devons nous entraider pour que cette représentation change», conclut-elle.
\end{abstract}

\section{Plus de femmes dans Wikipédia}

Depuis Genève, Natacha Rault, de l'association « Les sans pagEs » [3], nous raconte l'objectif de ce projet : réduire les biais de genre dans le Wikipédia francophone.

Concrètement, comment écrire sur une femme dans Wikipédia ? Il faut d'abord la trouver quelque part. Puis il faut vérifier qu'elle répond aux critères d'admissibilité (différents selon les langues). Ensuite, il faut dénicher des sources : articles de journaux, livres... Enfin, il existe des critères spécifiques pour les universitaires, qui doivent avoir reçu un prix scientifique reconnu internationalement, ou être considérés comme auteurs de référence. On constate que les femmes scientifiques sur Wikipédia sont davantage refusées que les hommes.

Natacha Rault appelle donc les journalistes à écrire davantage sur les femmes, pour pouvoir les inclure dans Wikipédia. Il faudrait aussi plus de contributrices pour améliorer la diversité des points de vue: Wikipédia est écrit à $80 \%$ par des hommes !

\section{Femmes de science en podcast}

Laurent Simon, fondateur de The Meta News, un média pour chercheurs et chercheuses créé en 2019, souligne que son média est lié aux chercheuses dès le début : "Nos abonnés étaient à $70 \%$ des abonnées. " Il a appris l'existence de l'effet Matilda ${ }^{(a)}$, découvert par l'historienne des sciences Margaret Rossiter : de nombreux apports de femmes aux sciences ont été occultés, comme celui de Rosalind Franklin sur l'ADN.

D'où l'idée, à The Meta News, de créer le podcast « Rendezvous avec Matilda ", qui en est à son quatrième épisode. Il invite des femmes de science actuelles, comme Élodie Chabrol, jeune biologiste qui s'est fait voler ses résultats, et dirige maintenant le festival international de vulgarisation "Pint of Science". "Mais c'est injuste que les femmes aient à raconter leur vie personnelle pour défendre la place des femmes en science : on ne demande pas cela aux hommes ", juge Laurent Simon.

\section{6 femmes de science... et bien plus}

Chaque jour en 2020, Alexis Verger a publié un tweet sur une femme scientifique, sur le compte @366portraits (fig. 3). Pourquoi ces portraits ? "Juste une idée personnelle ", se souvient le chercheur en biologie, qui regrette l'invisibilisation de femmes de science. Par exemple, "lorsqu'une découverte ou une équation porte un nom, on pense inconsciemment qu'il s'agit d'un homme."

Chaque portrait comporte un nom, une date de naissance, éventuellement de décès, la nationalité, et une description très succincte (format twitter oblige), ainsi qu'un lien internet pour aller plus loin. "Je ne fais que remonter l'information, le travail en amont est fait par des centaines de personnes" relativise Alexis Verger. Ses sources d'inspiration:Wikipédia, le livre Women in science de Rachel Ignotofsky, le projet "Beyond Curie" [4], ainsi que le site espagnol "Mujeres con ciencia" [5].

Le plus compliqué : faire des choix, forcément subjectifs. "J'ai parlé de femmes que je connais et que j'apprécie, j'ai adapté ma liste en fonction de l'actualité (coronavirus et vaccins), et j'ai diversifié les époques et les nationalités. Je voulais montrer que les femmes du monde entier font de la science. "

Alexis Verger a inspiré d'autres initiatives, par exemple @366femmes pour parler des femmes en sciences humaines, ou @365IndianWomxInSTEM pour les femmes scientifiques indiennes. La belle aventure continue : il veut aller jusqu'à au moins 500 portraits !

\section{Non au sexisme dans l'intelligence artificielle}

Aude Bernheim, chercheuse à l'Inserm, coautrice avec Flora Vincent du livre L'intelligence artificielle, pas sans elle, nous invite à nous pencher sur l'Intelligence artificielle (IA), présente partout. L'IA nous propose des films, aide à choisir qui aura des prêts, ou qui entrera dans quelle université. Mais qui la fait ? Essentiellement les hommes. Or, ses propositions peuvent être sexistes. Dans les logiciels de traduction, par exemple, "a scientist" sera traduit par « un scientifique " tandis que "a nurse" deviendra " une infirmière ». C'est également le cas pour certains choix de CV. Car les IA apprennent sur des bases de données, ellesmêmes biaisées.

Mais on peut aussi s'emparer de l'IA : diagnostic sur la représentation des femmes, mesure de temps de paroles, création automatique des pages Wikipédia de femmes... Autant d'outils fantastiques pour faire avancer l'égalité entre hommes et femmes.

De nombreux supports sur l'égalité dans l'IA sont libres de diffusion (en citant les auteurs et autrices) sur le site « iapassanselles » [6]. 


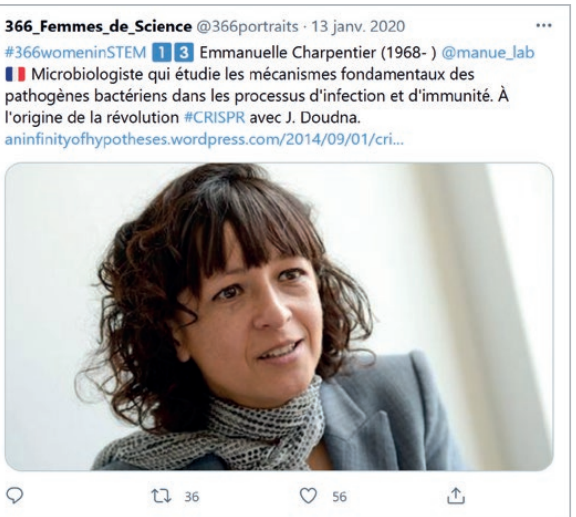

Ł๖ 3

O 56

3. Trois femmes scientifiques mises en avant sur le compte twitter @366portraits.

(a) La microbiologiste française Emmanuelle Charpentier, prix Nobel 2020 de chimie.

(b) La physicienne nigériane Francisca Nneka Okeke, lauréate du prix L'Oreal-Unesco pour les femmes et la science 2013

(c) La mathématicienne iranienne Maryam Mirzakhani, médaille Fields 2014

\section{Le rôle des organismes de recherche}

La visibilité des femmes de sciences se joue d'abord chez leur employeur. C'est le thème de la deuxième table ronde rassemblant Caroline Champenois, chercheuse CNRS et membre de la commission Femmes et Phpsique de la SFP, Liliana Cucu-Grosjean, coprésidente du comité parité de l'INRIA, et Marie-Ange Folacci, directrice de la communication du CEA.

Marie-Ange Folacci regrette qu'on " perde " des femmes qui, pourtant, ont fait de longues études. Leur donne-t-on les opportunités ? Les candidatures féminines reculent lorsque l'âge du recrutement augmente, comme actuellement. Liliana Cucu-Grosjean rappelle les biais de l'évaluation scientifique. Les jurys ne sont pas forcément paritaires dans les domaines très masculins (où les rares femmes sont sur-sollicitées). «En 2015, à l'INRIA, lorsqu'on a eu un jury plus paritaire, nous avons doublé le nombre de femmes admissibles, souligne-t-elle. Depuis qu'on affiche cette parité, on a davantage de candidatures féminines. "

La visibilité des femmes se joue aussi dans les congrès scientifiques. Il y a deux obstacles : le cout des déplacements et la garde des enfants. Liliana Cucu-Grosjean a demandé des financements sur ces deux points dans les congrès qu'elle coorganise ; cela a permis de faire passer la part de femmes de $5 \%$ à $30 \%$. La SFP, quant à elle, a rédigé une charte pour une représentation équitable des femmes lors des conférences, et la promeut auprès de différentes institutions. Que signifie " représentation équitable " ? Proportionnelle à la quantité de femmes dans le domaine? Liliana Cucu-Grosjean estime qu'il faut que les femmes soient visibles à hauteur du pourcentage de femmes que l'on vise et non de l'existant.

Il faut aussi former les managers hommes : au final, ce sont eux qui recrutent, souligne Marie-Ange Folacci. Caroline Champenois aimerait que le monde académique accepte de changer ses pratiques pour que les femmes s'y sentent mieux.

\section{Construire la confiance entre scientifiques et journalistes}

La dernière table ronde avait pour objet le lien entre scientifiques et journalistes. Elle réunissait Sabrina Krief, professeuse au Muséum national d'Histoire naturelle, Isabelle Collet, enseignante en sciences de l'éducation à l'université de Genève, et Karine Lacombe, cheffe du service maladies infectieuses à l'hôpital St Antoine à Paris.

Karine Lacombe rappelle qu'il existe beaucoup de femmes expertes. "Mais nous sommes plus facilement critiquées, de manière sexiste, si bien que beaucoup d'expertes préfèrent l'ombre à la lumière ". Pour Sabrina Krief, juste revenue d'Ouganda, les relations avec les journalistes se passent bien, ils aiment le côté exotique d'une femme dans la jungle étudiant les chimpanzés.

Karine Lacombe conseille aux expertes de prendre la parole et de la garder. «Plus nous serons nombreuses, plus la parole des expertes paraitra normale. Nous devons avoir confiance dans notre expertise, on a travaillé pour arriver à ce niveau. Avoir conscience de l'autocensure est un premier pas pour la combattre. " Isabelle Collet rappelle que les femmes ne naissent pas avec un sentiment d'autocensure : celui-ci se fabrique par une censure sociale régulière. On met en doute tout ce qu'elles disent ! Les commentaires sexistes après les articles des femmes le montrent bien. Inversement, " avoir des articles sur nous dans la presse nous donne une certaine assise vis-à-vis des collègues », souligne Isabelle Collet.

Cette confiance peut se construire avec le ou la journaliste, qui dit: "Je vous ai choisie pour votre compétence».
- www.culture.gouv.fr/presse/communiques-de-presse/, 15.09.2020, "Rapport sur la place des femmes dans les médias en temps de crise ». 2• https://expertes.fr/

3. https://sanspages.org

4. www.beyondcurie.com

5• https://mujeresconciencia.com

6• https://iapassanselles.wixsite.com/presentation 